\title{
Parameter Selection in a Combined Cycle Power Plant
}

\author{
Niklas Andersson $^{\mathrm{a}} \quad$ Johan $^{\mathrm{A} k e s s o n}{ }^{\mathrm{b}, \mathrm{c}} \quad$ Kilian Link $^{\mathrm{d}}$ \\ Stephanie Gallardo Yances ${ }^{\mathrm{d}}$ Karin Dietl $^{\mathrm{d}}$ Bernt Nilsson $^{\mathrm{a}}$ \\ a'Lund University, Department of Chemical Engineering, Lund, Sweden \\ ${ }^{b}$ Lund University, Department of Automatic Control, Lund, Sweden \\ ${ }^{\mathrm{c}}$ Modelon AB, Lund, Sweden \\ ${ }^{\mathrm{d}}$ Siemens AG, Erlangen, Germany
}

\begin{abstract}
A combined cycle power plant are modeled and considered for calibration. The dynamic model, aimed for start-up optimization, contains 64 candidate parameters for calibration. The number of parameter sets that can be created are huge and an algorithm called subset selection algorithm is used to reduce the number of parameter sets. The algorithm investigates the numerical properties of a calibration from a parameter Jacobean estimated from a simulation of the model with reasonably chosen parameter values. The calibrations were performed with a Levenberg-Marquardt algorithm considering the least squares of eight output signals. The parameter value with the best objective function value resulted in simulations in good compliance to the process dynamics. The subset selection algorithm effectively shows which parameters that are important and which parameters that can be left out.

Keywords: Combined Cycle Power Plants; Startup; Calibration; Parameter Selection
\end{abstract}

\section{Introduction}

Increasing environmental awareness has resulted in more demanding requirements. Energy supply is getting more attention and more and more wind turbines and solar power stations are built to adapt to a more environmentally friendly world. The challenge however is that the sun doesn't always shine and the wind doesn't always blow. Combined cycle power plants (CCPP) work as a good complement, because of its fast startup and shutdown time. Furthermore, the CCPPs have high thermal efficiency and are relatively environmentally friendly [1].

The market energy price fluctuates every day, which affects the profitability of the CCPPs. Because of this it is of importance to adapt to the market and be able to quickly start up and shut down the process. A quick start-up is important because energy is not produced until the gas turbine reaches full speed and is synchronized to the grid. However, this cannot be done too quickly because a rapid temperature change wears out sensible parts. The startup follows three phases: the first to accelerate the gas turbine to full speed, the second to increase the load of the gas turbine and the third to drive the steam to the steam turbine. The model of this work focuses on the second part which is the most critical during a startup. Sensible parts as the drum after the evaporator and the header of the superheater are important to model for a successful startup optimization. A model was therefore set up aimed for optimization of the startup, considering the temperature gradient in the sensible parts to estimate the tensions. The model has previously been used in optimizations $[2,3]$. The startup of combined cycle power plants has been optimized in several studies before $[4,5,6,7,8]$.

Accurate modelling of the CCPP is a difficult task, which if successful can cut expenses. This requires a good calibration of the model to make the discrepancy to the real process dynamics as small as possible. The main purpose of the calibration is to enable a valid model for optimization of startups. The parameter estimation is performed using a LevenbergMarquardt algorithm, which effectively uses the parameter Jacobean to find the optimum.

A model usually contains many parameter candidates for calibrations. Estimating many parameter simultaneously leads to ill-conditioned calibration problem due to dependency between the parameters, that make convergence bad and with wide confidence intervals as a result. A parameter-selection algorithm, 
called subset selection algorithm (SSA) is proposed that ranks the parameter by two properties, $\alpha$ and $\kappa$ [9]. The parameter $\alpha$ is correlated to the size of the confidence regions for a parameter set and $\kappa$ is a measure of how well-conditioned the parameter Jacobean for a parameter set is. The algorithm that significantly reduces the number of parameter sets to study, has recently been proven to work good for a model of a polyethylene plant [10].

\section{Theory}

\subsection{Differential algebraic equation systems}

The general non-linear index-1 differential algebraic equation (DAE) form is defined by

$$
\begin{aligned}
& \mathbf{0}=\mathbf{F}(\dot{\mathbf{x}}, \mathbf{x}, \mathbf{w}, \mathbf{u}, \mathbf{p}) \\
& \mathbf{y}=\mathbf{g}(\dot{\mathbf{x}}, \mathbf{x}, \mathbf{w}, \mathbf{u}, \mathbf{p}) \\
& \mathbf{x}\left(t_{0}\right)=\mathbf{x}_{0}
\end{aligned}
$$

where $\mathbf{x}, \mathbf{w}, \mathbf{u}$ and $\mathbf{p}$ are vectors denoting state and algebraic variables that describe the inputs and parameters of the model. In Eq 2, the output variables of the system that is subject to calibration are denoted $\mathbf{y}$. The initial state is defined by $\mathbf{x}_{0}$ and is expressed in Eq 3 . To solve the steady-state problem the state derivatives $\dot{\mathbf{x}}$ are set to 0 and $\mathbf{x}$ and $\mathbf{w}$ are solved to fulfill Eq 1 .

\subsection{Non-linear regression methods for differ- ential algebraic models}

Regression methods are roughly classified into two broad categories: gradient methods and direct-search methods [11]. The former depends on accurate parameter gradients, while the latter does not. Gradient methods include the Gauss-Newton Method, the steepest descent method and the LevenbergMarquardt method, while direct-search include the simplex method, differential evolution algorithms and pattern search $[12,13]$. The Gauss-Newton method gives the best results when gradients are available [14].

The Levenberg-Marquardt method is a more stable variant of the Gauss-Newton method [12] and can be used to solve the problems of estimating dynamic parameters, where the Newton step $\Delta \mathbf{p}$ is calculated from

$$
\left(\mathbf{J}^{T} \mathbf{W} \mathbf{J}+\delta \operatorname{diag}\left(\mathbf{J}^{T} \mathbf{W} \mathbf{J}\right)\right) \Delta \mathbf{p}=\mathbf{J}^{T} \mathbf{W}(\hat{\mathbf{y}}-\mathbf{y})
$$

where $\hat{\mathbf{y}}$ is the measurements for the output and $\delta$ is a Levenberg-Marquardt parameter, which controls the allowed step length and is updated in each iteration, based on the quality of the Newton step. The sensitivity matrix is defined as the parameter Jacobean matrix

$$
\mathbf{J}=\frac{d \mathbf{y}}{d \mathbf{p}}
$$

and was estimated with finite differences using the central approximation.

A single shooting approach is common to solve problems of estimating dynamic parameters [15]. It starts with a guess of the parameters. The dynamic model is then simulated, and the parameters are updated iteratively by a regression method, such as Levenberg-Marquardt method.

\subsection{Statistics}

Confidence regions are calculated to assess the quality of the parameter estimates. A confidence interval of $1-\beta$, with $n_{y}$ number of measurements and $n_{p}$ number of parameters, is estimated from

$$
\mathbf{p}^{*} \pm s_{p} T_{i n v}\left(\beta / 2, n_{y}-n_{p}\right)
$$

and means that there is a probability of $1-\beta$ that the true parameter lies within the estimated interval. Here, $\mathbf{p}^{*}$ is the calibrated parameters, $T_{i n v}$ is the inverse of the Student's T test and $s_{p}$ is the estimated standard error of the parameters defined as

$$
s_{p_{k}}=\sqrt{(\chi)_{k k}}, \quad \text { for } \mathrm{k}=1, \ldots, n_{p}
$$

where $\chi$ is the covariance matrix calculated as

$$
\chi=\sigma^{2}\left(\mathbf{J}^{T} \mathbf{W} \mathbf{J}\right)^{-1}
$$

where $\sigma^{2}$ is the error variance and can be estimated by

$$
\sigma^{2}=\frac{1}{n_{y}-n_{p}}|\hat{\mathbf{y}}-\mathbf{y}|^{2}
$$

and the diagonal weighting matrix, $\mathbf{W}$, used to scale the outputs, is defined as

$$
\mathbf{W}=\left(\begin{array}{cccc}
\hat{y}_{1}^{-2} & 0 & \cdots & 0 \\
0 & \hat{y}_{2}^{-2} & \cdots & 0 \\
\vdots & \vdots & \ddots & \vdots \\
0 & 0 & \cdots & \hat{y}_{n_{y}}^{-2}
\end{array}\right)
$$

\subsection{Subset selection algorithm}

The numerical properties of a parameter estimation can be estimated from the sensitivity matrix, J. An algorithm is suggested [9] that investigates parameter 
sets from a nominal operating point, and ranks the parameter sets according to two quantities: the condition number, $\kappa$, and the parameter selection score, $\alpha$. Here, $\kappa$ is defined as the ratio between the largest and smallest singular value of $\mathbf{J}$, and $\alpha$ is defined by $\alpha(\mathbf{p})=|v|$, where $v$ is the scaled standard error for the parameters

$$
v_{i}=s_{p i} / p_{i} \quad \text { for } \mathrm{i}=1, \ldots, n_{p}
$$

The quantities $\kappa$ and $\alpha$ are used to estimate the parameter dependencies and the uncertainties in the parameters. A low value of $\alpha$ shows that the estimated parameters have been accurately determined, while a low value of $\kappa$ shows that the calibration problem are well conditioned. Both $\alpha$ and the confidence interval are calculated from the standard error of the parameters and the quantities are closely related. A low value of $\alpha$ indicates narrow confidence intervals. The parameter $\kappa$ is a measure of how well the calibration problem is conditioned. Low values are preferable because it indicates that the parameters are independent of each other, while high values indicates a difficult calibration, where an inverse of the sensitivity matrix does not exist or can only be calculated with low accuracy.

Both $\alpha$ and $\kappa$ can be calculated without any calibration of the model, by setting some reasonable parameter values, referred to as nominal values, and determine the sensitivity matrix from a simulation. From the full sensitivity matrix, $\alpha$ and $\kappa$ can be calculated for every parameter subset. The sensitivity matrix for a parameter subset is created by taking the corresponding columns from the full sensitivity matrix.

\section{Methods}

\subsection{Modelling Languages and Tools}

The mathematical model has been implemented in Modelica [16], which is a high-level language for describing complex physical systems, supporting objectoriented concepts such as classes, components and inheritance. It can also encode textbook-style declarative equations. This modelling paradigm has significant advantages over the block-based paradigm that is often used in the context of physical modelling. In particular, acausal modelling systems do not require the user to solve the derivatives of a mathematical model. Instead, differential and algebraic equations may be mixed, which then typically results in a differential algebraic equation.

The calibrations in this paper have been performed using JModelica.org, which is a Modelica-based opensource platform targeted at dynamic optimization

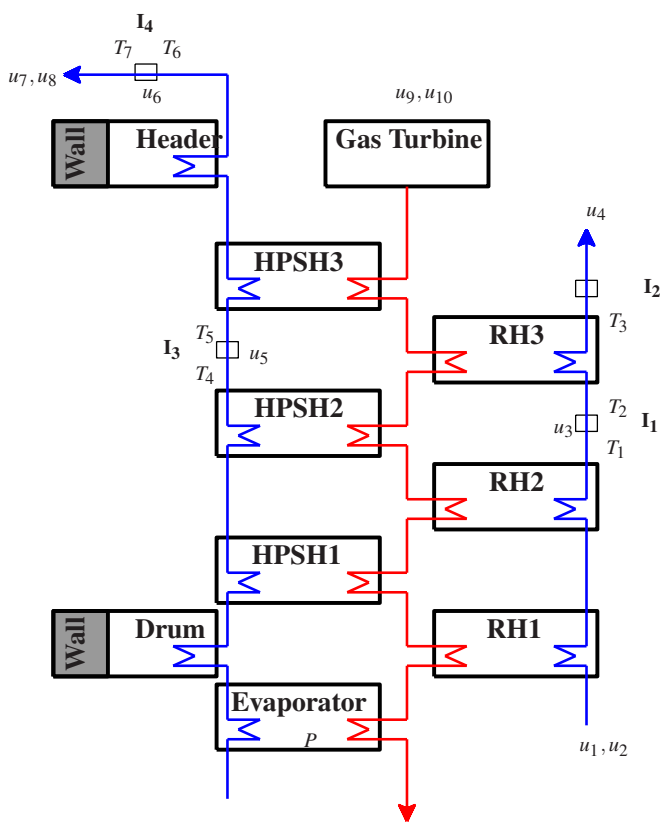

Figure 1: A schematic figure of the process.

[17]. The optimization is enabled by an extension to Modelica, Optimica, which strengthens its optimization capabilities by adding a small number of constructs. JModelica.org uses an interior point algorithm, IPOPT, to solve for feasible solutions, that fulfil the equation system[18]. Further, JModelica.org uses the Assimulo package [19], which interfaces the IDA solver from the Sundials suite [20].

To handle the high number of calibrations in this work in a reasonable time, a simple parallelization was performed. A simulation can only utilize one processor core, while it takes several simulations in every iteration of a calibration. By distributing the simulations with the python package subprocess, all eight processor cores could be utilized.

\subsection{Mathematical plant model}

A simple model scheme is found in Figure 1. The model has previously been used in startup optimization where it is described in more detail[2, 3]. The model, consisting of both differential and algebraic equations, has been derived from a combination of first principles and semi-empirical relations. It is focused on the heat recovery steam generator (HRSG) where the water side, indicated with blue arrows, are modeled by dynamic balance equations. The heat from the gas turbine (GT), shown with a red arrow, is statically modeled from the temperature $\left(u_{9}\right)$ and the mass flow $\left(u_{10}\right)$ of the GT. The water side is modeled as two different streams, one through the high pressure super- 


\begin{tabular}{cc}
\hline Notation & Description \\
\hline$u_{1}$ & RH inlet enthalpy \\
$u_{2}$ & IP mass flow \\
$u_{3}$ & water injection flow $I_{1}$ \\
$u_{4}$ & IP back pressure \\
$u_{5}$ & water injection $I_{3}$ \\
$u_{6}$ & water injection $I_{4}$ \\
$u_{7}$ & HP back pressure \\
$u_{8}$ & HP mass flow \\
$u_{9}$ & temperature GT \\
$u_{10}$ & mass flow GT \\
\hline
\end{tabular}

Table 1: A list of the inputs used.

heaters (HPSHs) and the other through the reheaters (RHs). The water is evaporated in the evaporator and going through the drum before it is superheated in three steps, HPSH1, HPSH2 and HPSH3. Finally the steam is led through the header and continues to the high pressure steam turbine. The drum and the header are similarly modeled as a volume, where the wall are subject to high stress during transients that needs to be constrained. The wall is spatially discretized so that the temperature gradient can be modeled, which is an indicator of the stress. The right blue line is going through the three reheaters $\mathrm{RH} 1, \mathrm{RH} 2$ and $\mathrm{RH} 3$ and continues to the intermediate pressure steam turbine. There are also four water injections modeled, shown in boxes in the Figure, where the first $I_{1}$ is located between RH2 and RH3 and $I_{2-4}$ are located after RH3, between HPSH 2 and HPSH3 and after the header, respectively. In the model there are also several valves to control the flow rates. The temperature sensors are also modeled to account for sensor lags.

The model is simulated with ten inputs following measurement data and are shown in Table 1. Three of the inputs are mass flows of the water injections, two describe the temperature and mass flow of the exhaust gas from the GT and five describe the state of the water on the HPSH and RH side. The mass flow of the exhaust gas from the GT are not measured directly, but calculated from balance equations. Eight objective signals are considered in the calibration and are shown in Table 2. The input and objective signals are also shown in Figure 1.

There are 64 potential parameters to calibrate in the model. The parameters are roughly divided in eight categories, see Table 3. Heat transfer coefficients are denoted as $k_{i n}$, describing the transfer between the exhaust gas and metal wall, $k_{\text {out }}$, describing the heat transfer between the metal wall and the cold water, and $k$, describing either the heat transfer in the sensors or the heat transfer in the metal walls of the header

\begin{tabular}{cc}
\hline Notation & Description \\
\hline$T_{1}$ & temperature before $I_{1}$ \\
$T_{2}$ & temperature after $I_{1}$ \\
$T_{3}$ & temperature before $I_{2}$ \\
$T_{4}$ & temperature before $I_{3}$ \\
$T_{5}$ & temperature after $I_{3}$ \\
$T_{6}$ & temperature before $I_{4}$ \\
$T_{7}$ & temperature after $I_{4}$ \\
$P$ & pressure evaporator \\
\hline
\end{tabular}

Table 2: A list of the objective signals used.

\begin{tabular}{|c|c|c|c|c|c|c|c|c|}
\hline & $k$ & $k_{\text {in }}$ & $k_{\text {out }}$ & $m_{\mathrm{H}_{2} \mathrm{O}}$ & $m_{F e}$ & V & cap & $k_{v}$ \\
\hline Header & 1 & & & & 7 & 15 & & \\
\hline Evaporator & & 3 & 17 & 2 & 20 & & & \\
\hline Drum & 10 & & & & 8 & 4 & & \\
\hline $\mathrm{SH}$ & & 9 & 5 & 18 & 21 & & & \\
\hline SH1 & & 28 & 22 & 56 & 62 & & & \\
\hline SH2 & & 29 & 23 & 57 & 63 & & & \\
\hline SH3 & & 30 & 24 & 58 & 64 & & & \\
\hline $\mathrm{RH}$ & & 12 & 6 & 19 & 11 & & & \\
\hline RH1 & & 34 & 25 & 59 & 31 & & & \\
\hline RH2 & & 35 & 26 & 60 & 32 & & & \\
\hline RH3 & & 36 & 27 & 61 & 33 & & & \\
\hline valves & & & & & & & & $\begin{array}{l}\mathbf{1 3}, 37,38, \\
39,40,41\end{array}$ \\
\hline sensors & $\begin{array}{c}\mathbf{1 4}, 42,43, \\
44,45,46, \\
47,48\end{array}$ & & & & & & $\begin{array}{c}\mathbf{1 6}, 49,50, \\
51,52,53, \\
54,55\end{array}$ & \\
\hline
\end{tabular}

Table 3: The parameter used in the SSA analysis. Merged parameters are indicated in bold.

and drum. There are two categories of masses, denoted as $m_{\mathrm{H}_{2} \mathrm{O}}$ for water volumes and $m_{\mathrm{Fe}}$ for iron walls. The last categories are the fluid volume $V$ for the header and drum and the heat capacity of the sensors, cap. Last category is $k_{v}$ that affects the dynamics of the valves, that is modeled with a constant pressure drop. There are seven sensors measuring the outputs $T_{1-7}$, each with two parameters and paired as $\{42,49\}$, $\{44,51\},\{43,50\},\{45,52\},\{47,54\},\{48,55\}$ and $\{46,53\}$.

Some parameters describe the same kind of parameter in different places of the model. For example, in the superheaters $\mathrm{SH} 1, \mathrm{SH} 2$ and $\mathrm{SH} 3$, the $k_{\text {out }}$ parameter is described with the parameters $22,23,24$ that has the same nominal values. A merged parameter is introduced that enables a reduction of parameters, which is important in calibration problems. For $k_{\text {out }}$ in the superheaters, the parameter 5 is a merged parameter. Setting this parameter means that the children parameters 22,23 and 24 gets the set value. There are 11 merged parameters in the analysis, where $k_{\text {in }}, k_{\text {out }}, m_{\mathrm{H}_{2} \mathrm{O}}$ and $m_{\mathrm{Fe}}$ are set by the parameters $9,5,18,21$ for the superheaters and the parameters $12,6,19,11$ for the reheaters, the parameter 13 sets all the other $k_{v}$ parameters and $k$ and cap for the temperature sensors are set with the parameters 14 and 16. A merged parameter can not be in the same parameter set as its children. 
Naturally, many of the parameters are highly correlated, such as $k_{\text {in }}$ and $k_{\text {out }}$. For convenience the parameter set $\left\{p_{1}, p_{2}, p_{3}\right\}$ is denoted as $p_{1,2,3}$

\section{Calibration methodology for large- scale systems}

\subsection{Calibration procedure}

The calibration is made by minimising the objective function described by a least square formulation of the error between the plant data and the model response. If all parameters are included in the calibrations, it leads to badly conditioned problems. The number of parameter sets that can be combined grows rapidly with the number of parameters. To reduce the number of parameters to estimate in the model a parameter selection algorithm called subset selection algorithm was used. Information from the sensitivity matrix is used to avoid ill-conditioned parameter estimations and to find parameter sets that can be determined with low parameter uncertainty.

The calibration procedure is solved by a single shooting procedure, where the model is simulated for every iteration of parameters. An attempt is made in each iteration to find the initial states for the updated parameters. The system simulation then proceeds during the whole start-up. The initial states are found by solving a steady-state problem, defined in Eqs. (1)-(3) and with $\dot{\mathbf{x}}=0$. The system is subsequently simulated, with the inputs $\mathbf{u}$ following the measurement data. The parameters can be updated by minimising the objective value defined as the weighted sum of squares of the residuals

$$
Q(\mathbf{p})=\sum_{i=1}^{n_{t}}\left(\hat{\mathbf{y}}_{i}-\mathbf{y}\left(t_{i}, \mathbf{p}\right)\right)^{T} \mathbf{W}\left(\hat{\mathbf{y}}_{i}-\mathbf{y}\left(t_{i}, \mathbf{p}\right)\right),
$$

where $n_{t}$ is the number of time points and $\mathbf{y}_{\mathbf{z}}\left(t_{i}, \mathbf{p}\right)$ is the model outputs from the simulation at time $t_{i}$. The calibration problem is solved iteratively by updating the parameters using the Levenberg-Marquardt algorithm, described in Section 2.2. The dynamic calibration is formulated as an optimization problem

$$
\begin{aligned}
& \min _{\mathbf{p}} Q(\mathbf{p}) \\
& \text { subject to Eqs. (1)-(3) } \\
& \mathbf{x}_{\text {min }} \leq \mathbf{x} \leq \mathbf{x}_{\text {max }} \\
& \mathbf{w}_{\text {min }} \leq \mathbf{w} \leq \mathbf{w}_{\text {max }} \\
& \mathbf{p}_{\text {min }} \leq \mathbf{p} \leq \mathbf{p}_{\text {max }}
\end{aligned}
$$

In this work, only one data set was used for calibration and no validation was performed. In a previous work, several calibration and validation data sets were used with a similar approach on another model, which showed good compliance [10].

\subsection{Reduction of parameter sets}

Models often have many potential parameters to calibrate, where many of the parameters are dependent of each other. If all parameters are included in the calibration it results in parameter Jacobeans that are highly ill-conditioned and a calibration that is impossible to solve. It is desirable to choose a subset of the parameters that are independent of each other, minimizes the objective function and with parameters that can be determined with good accuracy. The number of parameter combinations increase rapidly with the number of parameters and the exploration of all parameter sets is heavy computationally. An approach to reduce the parameter sets is suggested, where the $\kappa$ and $\alpha$ numbers of the SSA algorithm are used to rank the parameters. The selection method consists of two loops, the SSA loop and the calibration loop, each one consisting of three base parts: combination, evaluation and filter blocks, Figure 2. The blocks are defined as follows:

Combination is the process of taking an input population $\mathbb{P}_{\text {in }}$ that contains the $n_{\text {Pin }}$ parameter sets $\left\{\mathbf{p}_{i n}^{1}, \ldots, \mathbf{p}_{i n}^{n_{P i n}}\right\}$ and mixing it with all $n_{P 0}$ parameters $\mathbb{P}_{0}=\left\{p_{1}, \ldots p_{n_{P 0}}\right\}$ to create a new parameter set population $\mathbb{P}_{\text {out }}$ that contains parameter sets with one more parameter than the parameter sets of the input population. The input population is empty before the first iteration, and thus the output population will contain one parameter set for every parameter in $\mathbb{P}_{0} . \mathbb{P}_{\text {in }}$ is not empty before the next iteration, and thus the $\mathbf{p}_{i n}^{1}$ will enter the output population as $n_{P 0}$ parameter sets defined by $\left\{\left\{\mathbf{p}_{i n}^{1}, p_{1}\right\}, \ldots,\left\{\mathbf{p}_{i n}^{1}, p_{n_{P 0}}\right\}\right\}$, and the parameter sets $\mathbf{p}_{i n}^{2}, \ldots, \mathbf{p}_{\text {in }}^{n_{\text {Pin }}}$ will be combined in the same way. The same parameter set can be created from two different parameter sets in the input population, and thus an operation is carried out to remove all duplicates. The maximum number of parameter sets in the output population is $n_{P 0} n_{P i n}$, but this may be reduced when duplicates are removed. There are two combination blocks, Block 1 (SSA loop), where $\mathbb{P}_{\text {comb } 1}$ is created and Block 4 (calibration loop), where $\mathbb{P}_{\text {comb2 } 2}$ is created.

SSA Evaluation evaluates $\alpha$ and $\kappa$ values for each 
parameter set of the input population as defined in Section 2.4, and calculates a SSA score $\theta$, given by $\theta=\lg \alpha+\lg \kappa$, that is later used in the filter block to determine the best parameter sets in the SSA loop.

Calibration is the step where calibrations are made for all parameter sets in the input population, that consists of both $\mathbb{P}_{\text {cal } 1}$ and $\mathbb{P}_{\text {cal } 2 \text {. All parameter }}$ sets are calibrated and the objective value that measures the deviation between model and measurements is returned. The calibration step is the most computationally expensive step.

Filters are used to reduce the number of parameter sets, which otherwise would increase rapidly. There are three filter blocks, one in the SSA loop and two in the calibration loop. The filter block takes a population of parameter sets, a score that has been calculated to rank the parameter sets, and a cutoff that defines how many parameter sets should pass. In Block 2 and 5, $\theta$ is used as score and $n_{S S A 1}$ is used as cutoff for $\mathbb{P}_{S S A}, n_{\text {cal } 1}$ for $\mathbb{P}_{\text {cal } 1}$ and $n_{\text {cal } 2}$ for $\mathbb{P}_{\text {cal } 2}$. In Block $8, Q$ is used as score and $n_{Q}$ is used as cutoff. The choice of the cutoffs are arbitrarily, but should not be chosen too small for a good analysis.

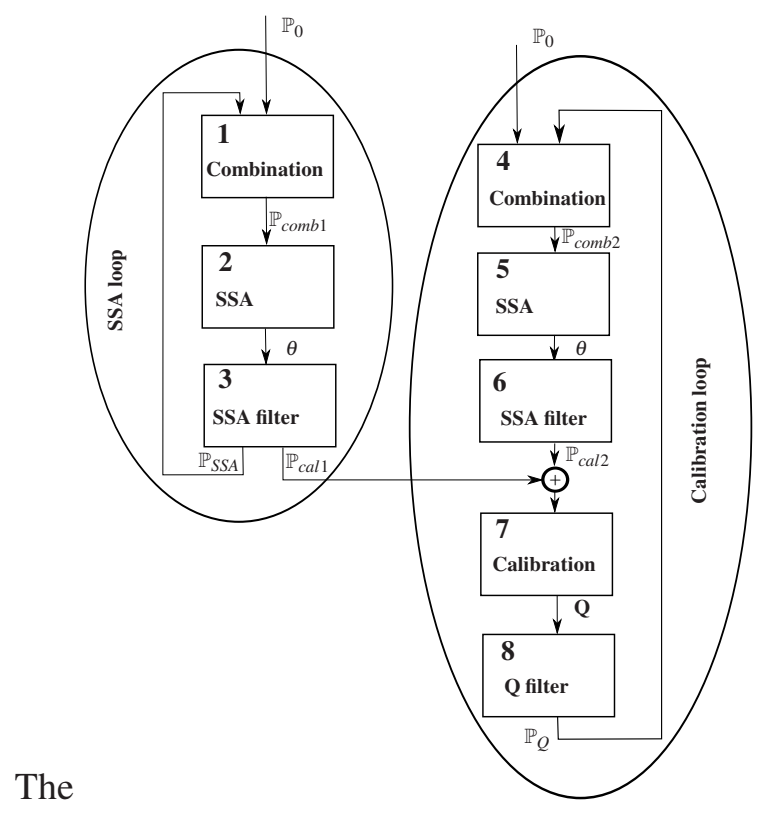

Figure 2: The SSA selection procedure used.

SSA evaluation is relatively cheap, but the number of parameter sets increase rapidly as $n_{p}$ increases. The number of parameter sets increases as the binomial coefficients $\left(\begin{array}{c}n_{P 0} \\ n_{p}\end{array}\right)$, which for $n_{P 0}=64$ are
$\{64,2016,41664,635376,7624512, \ldots\}$. Setting a filter cutoff limits the population that must be examined to $n_{S S A} n_{P 0}$ per iteration instead. The number of calibrations are dependent of $n_{P 0}$ and the filters $n_{c a l 1}$ and $n_{\text {ncal } 2}$. In the first iteration, $n_{\text {cal } 1}$ calibrations were performed and in the following rounds, $n_{\text {cal } 1}+n_{\text {cal2 }}$ calibrations are performed.

In this work, the cutoffs have been chosen to $n_{S S A}=$ $300, n_{\text {cal } 1}=5$ (10 in the first iteration), $n_{\text {cal } 2}=4$ and $n_{Q}=1$ in the work presented here. In this work the loops were iterated for parameter sets ranging from one parameter to seven parameters. The total number of calibrations performed is around five in the first iteration and nine in the rest, totally 59 calibrations.

\section{Calibration results}

\subsection{Calibration}

The calibration of the model was done for many parameter sets in the calibration loop of the SSA method. The parameter set with the best objective value with seven parameters, $p_{6,13,16,17,22,24,47}$, is called $C_{3}$ and is shown in Figure 3. The parameters in the set consist of four $k_{\text {out }}$ parameters for SH1, SH3 and all RHs, one valve parameter and two sensor parameters. No parameters for masses, volumes and heat transfer in walls and gas side are represented. The calibration reduces the objective function value from 1.85 with nominal parameter values to 0.585 and improves all eight objective signals. The largest improvements are for $T_{1}, T_{4}, T_{6}$ and $P$, with $72 \%, 70 \%, 87 \%$ and $74 \%$ reduction.

In Table 4 the calibrated parameters with confidence interval for the three calibrations $C_{1-3}$ are compared. The confidence intervals are narrow for all parameters, except for $p_{24}$ that is $k_{\text {out }}$ in SH3. This parameter affects mainly the objective $T_{6}$, which transient for the nominal parameter value are far below the measurements. To increase the temperature, the obtained parameter value is therefore very high, with the values 64.9, 79.3 and 79.5. The confidence intervals are almost as big as the parameter which is a very inaccurate parameter. The $\alpha$ and $\kappa$ values are also notably worse at the optimum.

\subsection{Parameter selection}

The SSA analysis was performed for the parameters and is shown in Figure 4. The evaluated parameter sets results in dot clouds that move upwards and to the right, the more parameters that are added. The dot 

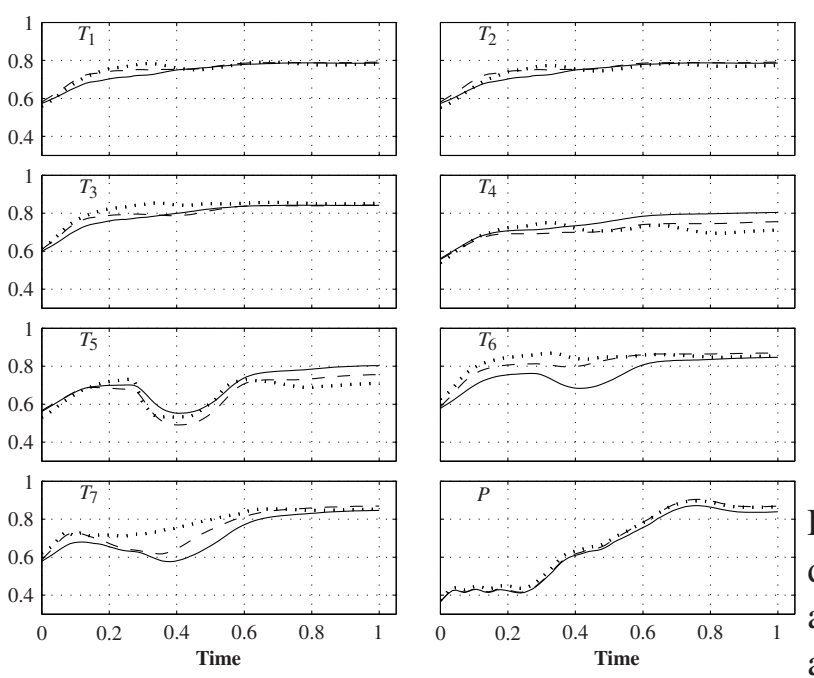

Figure 3: Simulation profiles from the best calibration for all states in the objective. The measurement data (dotted line) are shown together with the simulation with nominal (solid line) and optimal (dashed line) parameter values are shown in solid and dashed line.

\begin{tabular}{cccc}
\hline parameter & $C_{1}$ & $C_{2}$ & $C_{3}$ \\
\hline$p_{6}$ & $3.89 \pm 0.45$ & $4.28 \pm 0.62$ & $4.58 \pm 0.71$ \\
$p_{13}$ & $1.97 \pm 0.027$ & $2.17 \pm 0.043$ & $2.16 \pm 0.042$ \\
$p_{16}$ & & $0.92 \pm 0.023$ & $0.964 \pm 0.027$ \\
$p_{17}$ & & $0.66 \pm 0.020$ & $0.665 \pm 0.020$ \\
$p_{22}$ & & $0.38 \pm 0.014$ & $0.375 \pm 0.014$ \\
$p_{23}$ & $0.54 \pm 0.021$ & & \\
$p_{24}$ & $64.9 \pm 46.2$ & $79.3 \pm 78.7$ & $79.5 \pm 79.5$ \\
$p_{47}$ & $9.6 \pm 0.078$ & & $1.26 \pm 0.069$ \\
\hline
\end{tabular}

Table 4: Calibrated parameter values with a $95 \%$ confidence interval for calibrations $C_{1}$ and $C_{2}$. All parameters are scaled with the nominal parameter value.
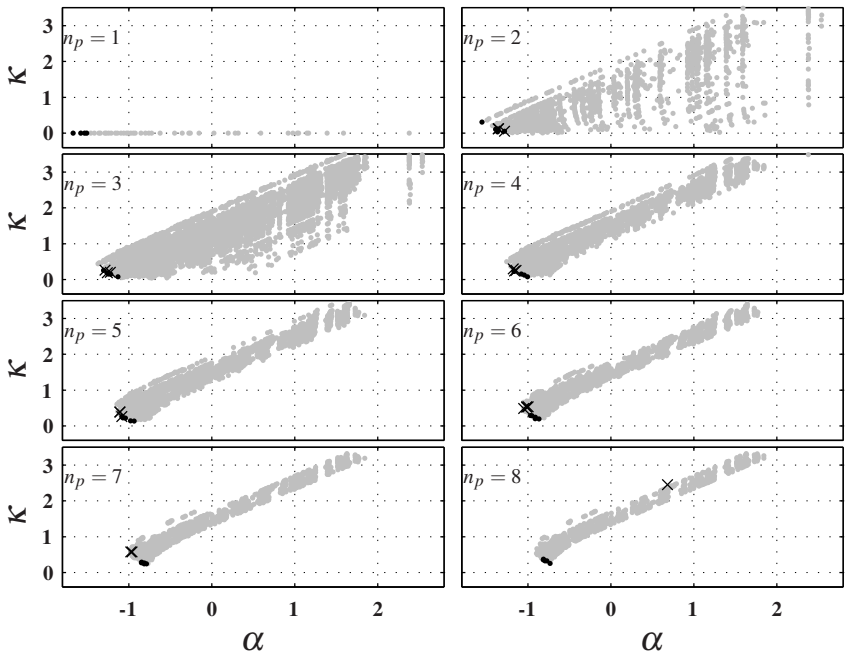

Figure 4: The SSA analysis for $n_{p}$ from 1 to 8 . The dot clouds move to the right and up when parameters are added. Parameter sets with best $\theta$ is marked as $(\bullet)$ and parameters from the best objective loop $(\times)$

clouds are created from the SSA loop of the SSA analysis, with 64 parameter sets for $n_{p}=1$ and $64 * 300$ parameter sets for $n_{p}$ from two to eight. The parameter sets with the lowest values of $\theta\left(\mathbb{P}_{c a l 1}\right)$ are shown with black dots and are located in the lower left corner in each figure. Those parameter sets were calibrated and the objective values are shown in Table 5. The objective value improves when parameters are added for $n_{p}$ from one to five. After that the calibration gets worse for $n_{p}$ equal to six and seven.

In the calibration loop of the SSA analysis, the best parameter sets were combined with new parameters to create $\mathbb{P}_{\text {cal } 2}$ that were calibrated and is presented in Table 6. Those parameter sets, marked with crosses are also located in the lower left corner for $n_{p}$ from two to seven. The objective values of this loop gets better for every iteration as new parameters are added to the best parameter set of the previous iteration until eight parameters are reached where the calibrations do not converge. Also the $\alpha$ and $\kappa$ values for those parameter sets are very bad.

In Table 5 there is 20 unique parameters $\{5,6,12$, $13,14,16,17,22,23,24,26,43,45,46,47,48,52,53$, $54,55\}$ where $\{5,6,12,13,14,16\}$ are merged parameters. Seven of the parameters, $\{5,6,17,22,23,24,26\}$ are $k_{\text {out }}$ parameters, while only one parameter, $\{12\}$ is a $k_{\text {in }}$ parameter, indicating that the parameters for heat transfer between the metal wall to the cold water have greater impact than the heat transfer between the exhaust gas and the metal wall. No drum or header parameter are visible in the best parameter 
Table 5: The calibration results from the left loop of the SSA analysis, sorted by $\theta .{ }^{*} C_{1} \dagger$ The $8^{\text {th }}$ value of $\theta$

\begin{tabular}{|c|c|c|c|c|}
\hline parameters & $\log _{10}(\alpha)$ & $\log _{10}(\kappa)$ & $\theta$ & obj \\
\hline$p_{5}$ & -1.87 & 0 & -1.87 & 1.77 \\
\hline$p_{14}$ & -1.65 & 0 & -1.65 & 1.77 \\
\hline$p_{16}$ & -1.65 & 0 & -1.65 & 1.77 \\
\hline$p_{17}$ & -1.56 & 0 & -1.56 & 1.85 \\
\hline$p_{22}$ & -1.55 & 0 & -1.55 & 1.85 \\
\hline$\dagger p_{24}$ & -1.46 & 0 & -1.46 & 1.03 \\
\hline$p_{6,23}$ & -1.51 & 0.0369 & -1.48 & 1.79 \\
\hline$p_{6,22}$ & -1.54 & 0.0947 & -1.45 & 1.79 \\
\hline$p_{6,17}$ & -1.54 & 0.109 & -1.43 & 1.8 \\
\hline$p_{5,14}$ & -1.7 & 0.31 & -1.39 & 1.73 \\
\hline$p_{5,16}$ & -1.7 & 0.31 & -1.39 & 1.73 \\
\hline$p_{6,23,24}$ & -1.67 & 0.152 & -1.52 & 0.832 \\
\hline$p_{6,17,24}$ & -1.69 & 0.19 & -1.5 & 0.895 \\
\hline$p_{6,24,47}$ & -1.64 & 0.147 & -1.49 & 0.899 \\
\hline$p_{6,24,54}$ & -1.64 & 0.148 & -1.49 & 0.899 \\
\hline$p_{6,22,24}$ & -1.68 & 0.192 & -1.49 & 0.808 \\
\hline$p_{6,17,24,47}$ & -1.6 & 0.225 & -1.38 & 0.895 \\
\hline$p_{6,17,24,54}$ & -1.6 & 0.225 & -1.38 & 0.895 \\
\hline$p_{13,26,46,48}$ & -1.44 & 0.0756 & -1.36 & 1.53 \\
\hline$p_{13,26,46,55}$ & -1.44 & 0.0756 & -1.36 & 1.58 \\
\hline$p_{13,26,48,53}$ & -1.44 & 0.0761 & -1.36 & 1.53 \\
\hline$* p_{6,13,23,24,47}$ & -1.5 & 0.227 & -1.28 & 0.675 \\
\hline$p_{6,13,23,24,54}$ & -1.5 & 0.227 & -1.28 & 0.675 \\
\hline$p_{12,13,46,48,54}$ & -1.41 & 0.144 & -1.27 & 1.53 \\
\hline$p_{12,13,46,54,55}$ & -1.41 & 0.144 & -1.27 & 1.58 \\
\hline$p_{12,13,46,47,48}$ & -1.41 & 0.144 & -1.27 & 1.53 \\
\hline$p_{12,13,45,46,48,54}$ & -1.34 & 0.202 & -1.14 & 1.52 \\
\hline$p_{12,13,45,46,54,55}$ & -1.34 & 0.202 & -1.14 & 1.57 \\
\hline$p_{12,13,45,48,53,54}$ & -1.34 & 0.202 & -1.14 & 1.52 \\
\hline$p_{12,13,45,53,54,55}$ & -1.34 & 0.202 & -1.14 & 1.56 \\
\hline$p_{12,13,45,46,47,48}$ & -1.34 & 0.203 & -1.14 & 1.52 \\
\hline$p_{13,26,43,45,46,48,54}$ & -1.27 & 0.264 & -1.01 & 1.49 \\
\hline$p_{13,26,43,45,46,54,55}$ & -1.27 & 0.264 & -1.01 & 1.54 \\
\hline$p_{13,26,43,45,48,53,54}$ & -1.27 & 0.264 & -1.01 & 1.49 \\
\hline$p_{13,26,43,45,53,54,55}$ & -1.27 & 0.264 & -1.01 & 1.54 \\
\hline$p_{13,26,43,46,48,52,54}$ & -1.27 & 0.264 & -1.01 & 1.5 \\
\hline
\end{tabular}

sets of the analysis. For the valves, only the merged parameter $\{13\}$ is visible in the tables. There are many sensor parameters visible in the result, namely $\{43,45,46,47,48,52,53,54,55\}$, corresponding to the sensors for $T_{1,4,5,6,7}$.

In Table 6 there is only 11 unique parameters $6,13,14,16,17,22,24,47,48,54,55$. All of those are not surprisingly also visible in Table 5, because they are partly derived from the best parameter sets of that table.

The parameter set with the lowest objective value for parameter sets with one parameter is $p_{24}$ with $Q=1.03$. This parameter is also visible for all of the best parameter sets, even though the confidence intervals are wide. The $\alpha$ and $\kappa$ values at the optimum were much worse at the optimum than for the nominal parameter values.

The parameter sets with $p_{47}$ and $p_{54}$ are replaceable in several places, for instance in $p_{6,22,24,47}$ and $p_{6,22,24,54}$ that give the same objective value. Both of
Table 6: The calibration results from the right loop of the SSA analysis, sorted by $\theta . * * C_{2} \quad * * * C_{3}$

\begin{tabular}{lllll}
\hline parameters & $\log _{10}(\alpha)$ & $\log _{10}(\kappa)$ & $\theta$ & obj \\
\hline & & & & \\
$p_{17,24}$ & -1.36 & 0.119 & -1.24 & 1.04 \\
$p_{24,47}$ & -1.28 & 0.0518 & -1.23 & 1.04 \\
$p_{24,54}$ & -1.28 & 0.0522 & -1.23 & 1.04 \\
$p_{22,24}$ & -1.36 & 0.125 & -1.23 & 1 \\
\hline & & & & \\
$p_{6,22,24}$ & -1.26 & 0.192 & -1.07 & 0.808 \\
$p_{16,22,24}$ & -1.29 & 0.263 & -1.02 & 0.935 \\
$p_{14,22,24}$ & -1.29 & 0.263 & -1.02 & 0.935 \\
$p_{22,24,47}$ & -1.22 & 0.204 & -1.02 & 1 \\
\hline & & & & \\
$p_{6,22,24,47}$ & -1.16 & 0.24 & -0.923 & 0.806 \\
$p_{6,22,24,54}$ & -1.16 & 0.24 & -0.923 & 0.806 \\
$p_{6,13,22,24}$ & -1.15 & 0.256 & -0.89 & 0.662 \\
$p_{6,17,22,24}$ & -1.18 & 0.299 & -0.88 & 0.773 \\
\hline & & & & \\
$p_{6,13,22,24,47}$ & -1.08 & 0.262 & -0.823 & 0.659 \\
$p_{6,13,22,24,54}$ & -1.08 & 0.262 & -0.822 & 0.663 \\
$p_{6,13,16,22,24}$ & -1.11 & 0.389 & -0.718 & 0.655 \\
$p_{6,13,14,22,24}$ & -1.11 & 0.39 & -0.717 & 0.741 \\
\hline & & & & \\
$* * p_{6,13,16,17,22,24}$ & -1.05 & 0.488 & -0.563 & 0.583 \\
$p_{6,13,16,22,24,47}$ & -1.02 & 0.528 & -0.487 & 0.655 \\
$p_{6,13,16,22,24,54}$ & -1.01 & 0.528 & -0.487 & 0.655 \\
$p_{6,13,16,22,24,48}$ & -0.999 & 0.536 & -0.463 & 0.666 \\
\hline & & & & \\
$* * * *$ & & & \\
$p_{6,13,16,17,22,24,47}$ & -0.975 & 0.576 & -0.398 & 0.585 \\
$p_{6,13,16,17,22,24,54}$ & -0.975 & 0.576 & -0.398 & 0.585 \\
$p_{6,13,16,17,22,24,48}$ & -0.961 & 0.579 & -0.383 & 0.598 \\
$p_{6,13,16,17,22,24,55}$ & -0.961 & 0.579 & -0.382 & 0.596 \\
\hline & & & & \\
\hline
\end{tabular}

those are sensor parameters for output $T_{5}$. Also the $T_{6}$ parameters $p_{48}$ and $p_{55}$ seem replaceable, apart from some small difference in objective value.

\section{Discussion and summary}

The objective function values became better when adding more parameters, but reached a point where adding of parameters made the calibrations too hard to solve. The best parameter set $\left(C_{3}\right)$ chose parameters from different parts of the model to minimize as many outputs as possible in the objective function.

The trend in the calibrations is that the parameter sets get harder to solve when more parameters are added and take more iterations. For eight parameters and more the calibrations fail to converge more often. Apart from that the calibrations are more complex when parameters are added, it is also harder to find independent parameters for a larger parameter set. Ill-conditioned calibration problems leads to parameter steps that make the simulations infeasible.

The analysis shows that the $k_{\text {out }}$ parameters occur more frequently than the $k_{\text {in }}$ parameters and indicates that the cold water side has greater impact of the model than the exhaust gas side. This is probably because the exhaust gas is only statically modeled in contrast to the cold water side. The analysis also shows that some parameters are replaceable, such as $p_{47}$ and $p_{54}$. Only 
one of the parameters are therefore needed for further analysis.

The merged parameters performed well in the analysis where six of the 11 merged parameters appeared in the best parameter sets. Merged parameters are effective for parameters that is expected to behave similarly and keeps the number of parameters in the calibration problem less.

The information to the SSA analysis is estimated from uncalibrated parameters but give a good indication about the best parameter sets considering the $\alpha$ and $\kappa$ values. The values are dependent of the parameter values and will obviously change for the calibrated parameter values, but hopefully not much. For most parameter sets, the $\alpha$ and $\kappa$ values stayed roughly the same, but for parameter sets including $p_{24}$ resulted in worse $\alpha$ and $\kappa$ values at the optimum. Still, the analysis highlights $p_{24}$ as a crucial parameter, that can decrease the objective function value the most, but with very wide confidence intervals as a result. A further analysis is required to understand this behavior.

Both the SSA and calibration loop of the analysis is dependent of the cutoff numbers for good performance. The calibration numbers were consciously set to low numbers, because of long calibration times that were performed on a single computer. The numbers can be set higher for a more thorough analysis if time or a computer cluster is available. The result shown here proves that satisfactory calibration results can be reached even with low cutoff numbers.

The parameter estimation results are in good compliance to the process dynamics. The subset selection algorithm effectively shows which parameters that are important and which parameters that can be left out. Considering the few number of calibrations that were performed, the result is satisfactory.

\section{References}

[1] Kehlhofer, R., Warner, J., Nielsen, H., Backmann, R., Combined-Cycle Gas and Steam Turbine Power Plants. ISBN: 0-87814-736-5, second edition, PennWell Publishing Com-pany, Tulsa, Oklahoma, USA, 1999.

[2] Lind, A., Sällberg, E., Velut, S., Åkesson, J., Gallardo Yances, S., Link, K. Sep. 2012. Start-up Optimization of a Combined Cycle Power Plant, 9th International Modelica Conference. Munich, Germany.
[3] Lind, A., Sällberg, E. Optimization of the Startup Procedure of a Combined Cycle Power Plant, Master's Thesis, Lund University, Department of Automatic Control, 2012

[4] Casella, F., Pretolani, F. Fast Start-up of a Combined-Cycle Power Plant: A Simulation Study with Modelica. In: Modelica Conference pp. 3-10, Vienna, Austria, 2006.

[5] Casella, F. Leva, A. Modelica open library for power plant simulation: design and experimental validation. In: Proceedings of 3rd International Modelica Conference, pp. 41-50. Linköping, Sweden, 2003.

[6] Casella, F., Farina, M., Righetti, F., Scattolini, R., Faille, D., Davelaar, F., Tica, A., Gueguen, H. Dumur, D. An optimization procedure of the start-up of combined cycle power plants.In: 18th IFAC World Congress, pp. 7043-7048. Milano, Italy, 2011.

[7] Casella, F., Donida, F., Åkesson, J. Objectoriented modeling and optimal control: a case study in power plant start-up. In: 18th IFAC World Congress, pp. 9549-9554. Milano, Italy, 2011.

[8] Shirakawa, M., Nakamoto, M., Hosaka, S. Dynamic simulation and optimization of start-up processes in combined cycle power plants. In: JSME International Journal, vol. 48 (1), pp. 122128, 2005.

[9] Cintrón-Arias, A., Banks, H. T., Capaldi, A., Lloyd, A. L., 2009. A Sensitivity Matrix Based Methodology for Inverse Problem Formulation. Journal of Inverse and Ill-Posed Problems, 17(6), 545-564.

[10] Andersson N., Larsson, P.-O., Åkesson, J., Carlsson, N., Skålén, S. Nilsson, B. Parameter selection in the parameter estimation of grade transitions in a polyethylene plant. submitted for publication.

[11] Edgar, R., Himmelblau, D., 1988. Optimization of Chemical Processes, 1st Edition. McGrawHill, New York, NY.

[12] Englezos, P., Kalogerakis, N., 2000. Applied parameter estimation for chemical engineers, 1st Edition. CRC Press. 
[13] Storn, K., Price, R., Lampinen, J. 2005 Differential Evolution - A Practical Approach to Global Optimization, Springer-Verlag, Berlin.

[14] Comparison of gradient methods for the solution of nonlinear parameter estimation problems. SIAM Journal on Numerical Analysis 7 (1), 157-186.

URL http://www . jstor.org/stable/2949590

[15] Vassiliadis, V., 1993. Computational solution of dynamic optimization problem with general differential-algebraic constraints. Ph.D. thesis, Imperial Collage, London, UK

[16] The Modelica Association, 2011. The Modelica Association Home Page. http://www.modelica.org

[17] Åkesson, J., and Årzén, K.-E., Gäfvert, M., Bergdahl, T., Tummescheit, H., nov 2010. Modeling and Optimization with Optimica and JModelica.org-Languages and Tools for solving large-scale dynamic optimization problem. Computers and Chemical Engineering 34 (11), 1737-1749.

[18] Wächter, A., Biegler, L. T., 2006. On the implementation of an interior-point filter linesearch algorithm for large-scale nonlinear programming. Mathematical Programming 106 (1), $25-58$

[19] Andersson, C., Andreasson, J., Führer, C., ^ kesson, J., 2012. A workbench for multibody systems ode and dae solvers. In: 2nd Joint International Conference on Multibody System Dynamics. Stuttgart, Germany.

[20] Hindmarsh, A. C., Brown, P. N., Grant, K. E., Lee, S. L., Serban, R., Shumaker, D. E., Woodward, C. S., September 2005. Sundials: Suite of nonlinear and differential/algebraic equation solvers. ACM Trans. Math. Softw. 31, 363-396

URL http://doi .acm.org/10.1145/1089014.1089020 